\title{
Exclusive Strategy for Generalization Algorithms in Micro-data Disclosure
}

\author{
Lei Zhang $^{1}$, Lingyu Wang ${ }^{2}$, Sushil Jajodia ${ }^{1}$, and Alexander Brodsky ${ }^{1}$ \\ ${ }^{1}$ Center for Secure Information Systems \\ George Mason University \\ Fairfax, VA 22030, USA \\ \{lzhang8, jajodia, brodsky\}@gmu.edu \\ ${ }^{2}$ Concordia Institute for Information Systems Engineering \\ Concordia University \\ Montreal, QC H3G 1M8, Canada \\ wang@ciise.concordia.ca
}

\begin{abstract}
When generalization algorithms are known to the public, an adversary can obtain a more precise estimation of the secret table than what can be deduced from the disclosed generalization result. Therefore, whether a generalization algorithm can satisfy a privacy property should be judged based on such an estimation. In this paper, we show that the computation of the estimation is inherently a recursive process that exhibits a high complexity when generalization algorithms take a straightforward inclusive strategy. To facilitate the design of more efficient generalization algorithms, we suggest an alternative exclusive strategy, which adopts a seemingly drastic approach to eliminate the need for recursion. Surprisingly, the data utility of the two strategies are actually not comparable and the exclusive strategy can provide better data utility in certain cases.
\end{abstract}

\section{Introduction}

The dissemination and sharing of information has become increasingly important to our society. However, such efforts may be hampered by the lack of security and privacy guarantees. For example, when a healthcare organization releases tables of diagnosis information, explicit identifiers such as names will be removed. However, an adversary may still identify a patient from the released table if, say, the combination of the patient's race, date of birth, and Zip code can be linked to a unique record in a publicly available voter list [20|24 25].

Existing solutions to the micro-data release problem are largely based on randomization or generalization. This paper considers generalization techniques. At an abstract level, a micro-data table can be considered as a mapping between quasi-identifiers (for example, the combination of race, date of birth, and Zip code) and sensitive values (such as diagnosis result). A generalization can be regarded as a partition on this mapping, which divides quasi-identifiers and corresponding sensitive values into disjoint groups. By hiding the detailed mapping inside each group, each quasi-identifier is blended with others in the same group. The amount of privacy protection achieved through such a generalization can be measured under various privacy properties, such as $l$-diversity[2].

V. Atluri (Ed.): DAS 2008, LNCS 5094, pp. 190-204, 2008.

(c) IFIP International Federation for Information Processing 2008 
However, a major limitation of most existing solutions is to assume a disclosed table to be the only source of information available to an adversary. Unfortunately, this is not always the case. An adversary usually knows the fact that a generalization algorithm will maximize the utility function in addition to satisfying the privacy property (relying on the secrecy of such information is an example of security by obscurity). As recently pointed out in [30], this extra knowledge may allow the adversary to obtain a more precise estimation of the secret table, on which the privacy property may no longer be satisfied. An apparent solution is to anticipate what the adversary will do, that is to estimate the secret table based on both the disclosed table and public knowledge about the generalization algorithm. Once the estimation is obtained, the privacy property can be evaluated to decide the safety of the generalization algorithm.

In this paper, we study the computation of an adversary's estimation of the secret table, which can be modeled as a set of possible instances of the unknown secret table, namely, disclosure set. We show that a given sequence of generalization functions can be combined into different strategies in releasing generalized tables. We first consider generalization algorithms designed under a straightforward inclusive strategy. We show that the computation of disclosure sets under the inclusive strategy is inherently a recursive process and exhibits a high complexity. To facilitate the design of efficient generalization algorithms, we then suggest an alternative exclusive strategy, which adopts a seemingly more drastic approach to generalization in order to avoid the need for a recursive process. Surprisingly, we show that the data utility of those two strategies are actually incomparable, and the exclusive strategy can provide better data utility in certain cases. First of all, we motivate further discussions with an example.

Motivating Example. Table1 1 shows our running example as a table containing patient information. The table has three attributes: Name, Age, and Patient's Condition. The attribute Name is an identifier. We assume the Age attribute forms a quasi-identifier, and the Condition attribute is sensitive.

Table 2 shows three possible generalizations, $G_{1}, G_{2}$ and $G_{3}$, together with the original table, in an abstract way. We denote as $I D$ the identifier (that is, Name), $Q I$ the quasi-identifier (that is, Age), and $S$ the sensitive attribute (that is, Condition). Each generalization $G_{1}, G_{2}$ and $G_{3}$ includes a group quasi-identifier $Q I_{i}(i=1,2,3)$ and the sensitive attribute $S$ (notice the identifier $I D$ has been removed). For simplicity, we omit the details of each group quasi-identifier and sensitive value in the remainder of this paper.

We assume the generalization algorithm to be public knowledge. This knowledge has several aspects. First, the generalization algorithm defines a sequence of generalization functions sorted in a non-increasing order of data utility. In Table 2 the three generalizations are results of applying $g_{1}, g_{2}$, and $g_{3}$ to the original table $G_{0}$, respectively. We assume the three generalizations have non-increasing data utility (for example, average group size). The assumption of given aggregation functions is a common practice of most existing generalization techniques. Although the number of possible generalization functions may grow quickly, say, in the number of attributes, the issue of choosing suitable aggregation functions among all possibilities is beyond the scope of this paper. Our assumption of non-increasing utility in the sequence of functions is also a common practice, and also notice that functions with equal or incomparable utilities 
Table 1. An Example of Patient Information Table

\begin{tabular}{|c|c|c|}
\hline Name & Age & Condition \\
\hline Alice & 21 & flu \\
\hline Bob & 27 & tracheitis \\
\hline Clark & 31 & pneumonia \\
\hline Diana & 36 & tracheitis \\
\hline Ellen & 43 & gastritis \\
\hline Fen & 49 & gastritis \\
\hline George & 52 & cancer \\
\hline Henry & 58 & enteritis \\
\hline Ian & 63 & cancer \\
\hline Jason & 67 & heart disease \\
\hline
\end{tabular}

Table 2. An Example of Three Generalization Functions

\begin{tabular}{|c|c|c|c|c|c|c|c|c|}
\hline \multicolumn{3}{|c|}{ Original Table $G_{0}$} & \multicolumn{2}{|c|}{ Generalization $G_{1}$} & \multicolumn{2}{|c|}{ Generalization $G_{2}$} & \multicolumn{2}{|c|}{ Generalization $G_{3}$} \\
\hline$I D$ & $Q I$ & $S$ & $Q I_{1}$ & $S$ & $Q I_{2}$ & $S$ & $Q I_{3}$ & $S$ \\
\hline$A$ & $g_{0}^{\perp}(21)$ & $c_{1}$ & $g_{1}^{\perp}$ & $c_{1}$ & $g_{2}^{\perp}$ & $c_{1}$ & $g_{3}^{1}$ & $c_{1}$ \\
\hline $\bar{B}$ & $g_{0}^{2}(27)$ & $c_{2}$ & $(20 \sim 29)$ & $c_{2}$ & $(20 \sim 29)$ & $c_{2}$ & $(20 \sim 34)$ & $c_{2}$ \\
\hline$C$ & $g_{0}^{3}(31)$ & $c_{3}$ & $g_{1}^{2}$ & $c_{3}$ & $\overline{g_{2}^{2}}$ & $c_{3}$ & & $c_{3}$ \\
\hline$D$ & $g_{0}^{4}(36)$ & $c_{2}$ & $(30 \sim 39)$ & $c_{2}$ & $0 \sim 44)$ & $c_{2}$ & 93 & $c_{2}$ \\
\hline$E$ & $g_{0}^{5}(43)$ & $c_{4}$ & $g_{1}^{3}$ & $c_{4}$ & & $c_{4}$ & $(35 \sim 54)$ & $c_{4}$ \\
\hline$F$ & $g_{0}^{6}(49)$ & $c_{4}$ & $(40 \sim 49)$ & $c_{4}$ & $g_{2}^{3}$ & $c_{4}$ & & $c_{4}$ \\
\hline$G$ & $g_{0}^{7}(52)$ & $c_{6}$ & $g_{1}^{4}$ & $c_{6}$ & $(45 \sim 59)$ & $c_{6}$ & & $c_{6}$ \\
\hline$H$ & $g_{0}^{8}(58)$ & $c_{5}$ & $(50 \sim 59)$ & $c_{5}$ & & $c_{5}$ & $g_{3}^{3}$ & $c_{5}$ \\
\hline$I$ & $g_{0}^{9}(63)$ & $c_{6}$ & $g_{1}^{5}$ & $c_{6}$ & $g_{2}^{4}$ & $c_{6}$ & $(55 \sim 69)$ & $c_{6}$ \\
\hline$J$ & $g_{1}^{10}(67)$ & $c_{7}$ & $(60 \sim 69)$ & $c_{7}$ & $(60 \sim 69)$ & $c_{7}$ & & $c_{7}$ \\
\hline
\end{tabular}

can be treated in the same way in our discussions. Second, the generalization algorithm defines a privacy property. In this paper, we consider a particular privacy property, namely, recursive $(2,2)$-diversity (basically, among all possible sensitive values that any record can take, the highest ratio of any value $X$, denoted as $r_{D S}(X)$, should satisfy $r_{D S}(X)<2\left(1-r_{D S}(X)\right)$, or equivalently, $\left.r_{D S}(X)<2 / 3\right)$ [2]. Third, the generalization algorithm applies the sequence of generalization functions to the original table, and returns the first generalization on which the privacy property evaluates to true. Clearly, this approach aims to maximize the data utility while satisfying the privacy property.

However, the above knowledge about the generalization algorithm may allow an adversary to deduce more information than what is directly disclosed in the generalization. For example, in Table 2 consider two cases. First, suppose an adversary does not know about the generalization algorithm, but only sees the second generalization $G_{2}$. In guessing the original table $G_{0}$, the adversary cannot discriminate the sensitive values in each group with respect to their association with each ID. For example, the ID $A$ can be associated with either $c_{1}$ or $c_{2}$ in the group $g_{2}^{1}$. Therefore, to the adversary, all tables obtained by permuting the sensitive values within each group can potentially be 
the original table. Second, suppose an adversary knows about the generalization algorithm in addition to seeing $G_{2}$. The adversary can then deduce that $G_{1}$ must not satisfy the recursive $(2,2)$-diversity because otherwise $G_{1}$ will be returned instead of $G_{2}$ due to better data utility. Although the adversary cannot see $G_{1}$ (more precisely, the sensitive values of $G_{1}$ ), based on the relationship between the groups in $G_{1}$ and $G_{2}$, he/she can still conclude that both $E$ and $F$ must be associated with $c_{4}$ in the original table. Clearly, between the above two cases, the recursive $(2,2)$-diversity is satisfied in the first but not satisfied in the second.

The above example shows that it is insufficient to evaluate a privacy property based on a generalization itself when the generalization algorithm is publicly known. Unfortunately, this is indeed the approach adopted by most existing generalization algorithms. Those algorithms may thus produce results that actually violate the given privacy property (we say such algorithms are unsafe). To develop safe generalization algorithms, a critical question is: What exactly can an adversary deduce about the original table, when he/she knows about the generalization algorithm? In this paper, we first show how to exactly compute an adversary's knowledge about the original table, namely, the disclosure set. Second, as a consequent, we obtain a safe version of the traditional approach to generalization by evaluating the privacy property on the disclosure set instead of the generalization. Later in this paper, we shall show that by applying the safe version of the generalization algorithm to the above example, we would reach the counter-intuitive conclusion that neither $G_{2}$ nor $G_{3}$ can be safely disclosed.

Organization. The remainder of the paper is organized as follows. Section 2 shows how to compute a disclosure set and reveals the inherent complexity of such a process. Section 3 introduces the exclusive strategy and studies the complexity and data utility of the corresponding generalization algorithms. Section 4 reviews related work. Section 5 finally concludes the paper.

\section{Computing Disclosure Sets under the Inclusive Strategy}

Section 2.1 first introduces the concept of disclosure set. Section 2.2 then studies the computation of disclosure sets under the inclusive strategy.

\subsection{Disclosure Set}

We consider the following micro-data disclosure problem. An original table $G_{0}(I D$, $Q I, S)$ is given where $I D, Q I$, and $S$ denote the identifier attribute, quasi-identifier attribute(s), and sensitive attribute, respectively. A generalization algorithm $\mathbb{G}$ is given, which defines a sequence of generalization functions $g_{1}, g_{2}, \ldots, g_{n}$. The algorithm $\mathbb{G}$ applies each $g_{i}$ in the given order to $G_{0}$ to obtain a generalization $G_{i}\left(Q I_{i}, S\right)$ where $Q I_{i}$ is the group quasi-identifier attribute. We assume the last generalization function $g_{n}$ always yields an empty set, indicating that nothing should be disclosed. The algorithm $\mathbb{G}$ always returns a generalization $G_{i}$ that satisfies a given privacy property $C H K$.

The above discussion, however, does not address a critical issue, that is how the given privacy property $C H K$ should be evaluated when a generalization $G_{i}$ is to be 
disclosed. Generally, $C H K$ should be evaluated based on an adversary's knowledge about the original table $G_{0}$. Such knowledge can be characterized as follows. The adversary attempts to guess $G_{0}$ based on the disclosed generalization $G_{i}$ and the public information about the generalization algorithm $\mathbb{G}$. Any table that contradicts the information available to the adversary will be eliminated. The adversary will end up with a set of possible instances, which represents the best guess the adversary can make about $G_{0}$, namely, his/her knowledge about $G_{0}$. We call such a set the disclosure set corresponding to the generalization function $g_{i}$, denoted as $D S_{i}$. Clearly, the privacy property $C H K$ should be evaluated on $D S_{i}$, when the generalization $G_{i}$ is to be disclosed.

If a disclosed generalization is the only source of information available to the adversary, then the disclosure set $D S_{i}$ is simply the collection of tables to which applying $g_{i}$ will yield the generalization $G_{i}$. Such a collection of tables can be obtained by fixing an order on the attribute $I D$ and $Q I$ while permuting the sensitive values within each group of $G_{i}$. We denote the collection of tables as $P E R\left(G_{i}\right)$. For example, in Table 2 $\operatorname{PER}\left(G_{1}\right)$ includes $2 \times 2 \times 1 \times 2=16$ tables since every group except $g_{1}^{3}$ has two permutations.

As illustrated in Section 1 we cannot simply evaluate $C H K$ on $\operatorname{PER}\left(G_{i}\right)$ when the generalization algorithm $\mathbb{G}$ is publicly known. The reason is that an adversary may eliminate some possible instances from $\operatorname{PER}\left(G_{i}\right)$ due to a conflict with the fact that $G_{i}$ is disclosed by $\mathbb{G}$. More precisely, the adversary can apply $\mathbb{G}$ to each possible instance in $\operatorname{PER}\left(G_{i}\right)$. If $\mathbb{G}$ returns any $G_{j}(j<i)$, then the adversary knows this possible instance cannot be the original table $G_{0}$, and hence it should not be included in $D S_{i}$. In this way, the adversary can derive $D S_{i}$ as a subset of $P E R\left(G_{i}\right)$.

Example 1. In Table 2 $D S_{1}$ is simply $P E R\left(G_{1}\right)$ because generally the original table will not satisfy $C H K$, so the adversary cannot eliminate any instance from $\operatorname{PER}\left(G_{1}\right)$. Although we shall delay the computation of $D S_{2}$, we can see that any possible instance in $\operatorname{PER}\left(G_{2}\right)$ that has two different sensitive values associated with the ID $E$ and $F$ will cause $\mathbb{G}$ to return $G_{1}$ instead of $G_{2}$, and thus will not be included in $D S_{2}$.

We formalize the concept of disclosure set in Definition 1

Definition 1. The disclosure set $D S_{i}$ corresponding to a generalization $G_{i}$ is a set of possible instances that satisfy

- $D S_{i} \subseteq \operatorname{PER}\left(G_{i}\right)$

- $\forall X \in D S_{i}$ the generalization algorithm $\mathbb{G}$ will not return $G_{j}$ for any $j<i$.

\subsection{The Computation of Disclosure Set}

Table 3 shows two algorithms. $\mathbb{G}$ on the left-hand side is a generalization algorithm and $\mathbb{D S}$ on the right-hand side is an algorithm for computing the disclosure set of a given generalization. $\mathbb{G}$ simply returns the first generalization $G_{i}$ whose disclosure set (computed by the other algorithm $\mathbb{D S}$ ) satisfies a given privacy property $C H K$. On the other hand, $\mathbb{D} \mathbb{S}$ computes the disclosure set of $G_{i}$ by eliminating from $\operatorname{PER}\left(G_{i}\right)$ any instance $X$ for which the algorithm $\mathbb{G}$ returns a generalization that appears before $G_{i}$. 
Table 3. Algorithms $\mathbb{G}$ and $\mathbb{D S}$

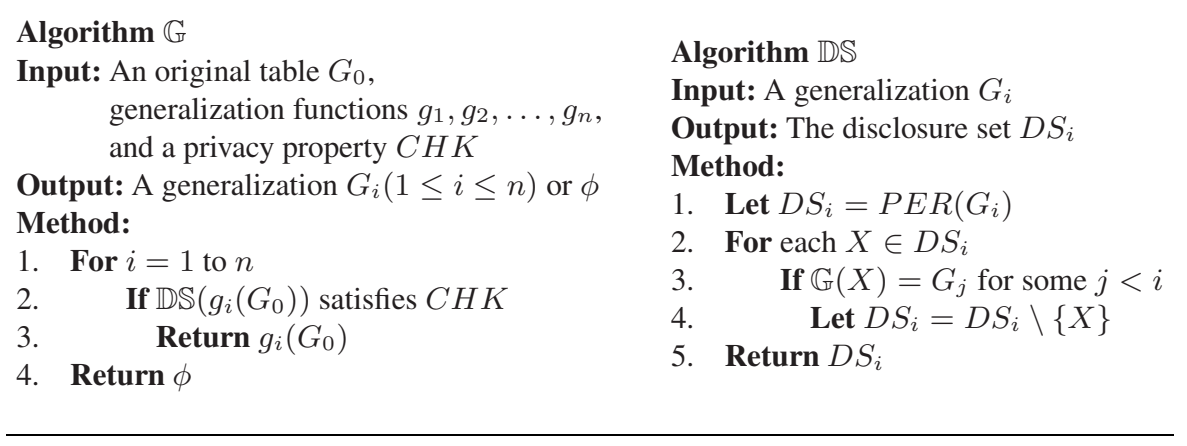

The algorithms in Table 3 show that the computation of disclosure sets is inherently a recursive process. In the algorithm $\mathbb{D} \mathbb{S}$, to compute the disclosure set of a generalization $G_{i}$, we must test every possible instance $X$ in $\operatorname{PER}\left(G_{i}\right)$ to determine whether $X$ should be included in $D S_{i}$. More specifically, we first assume $X$ to be the original table, then we apply the generalization algorithm $\mathbb{G}$. Each call to $\mathbb{G}$ will then involve $i-1$ calls to the algorithm $\mathbb{D} \mathbb{S}$ for computing the disclosure set of the generalizations $g_{j}(X)(j=1,2, \ldots, i-1)$ (each such computation will again involve multiple calls to the generalization algorithm). One subtlety here is that we are actually using a modified version of $\mathbb{G}$ since it only uses the first $i-1$ generalization functions. This is in accordance with Definition [1] and it also guarantees the recursive process to always terminate.

Example 2. In Table 2] to compute $D S_{2}$, the algorithm $\mathbb{D S}$ will call the algorithm $\mathbb{G}$ with each of the possible instances as the input. In this simple case, only $g_{1}$ is applied to each instance, and $D S_{1}$ is simply equal to $\operatorname{EXP}\left(G_{1}\right)$. Clearly, for any instance in which $E$ and $F$ are not both associated with $c_{4}$, the disclosure set $D S_{1}$ will satisfy the $(2,2)$-diversity, and hence the instance is not included in $D S_{2}$. On the other hand, all instances in which both $E$ and $F$ are associated with $c_{4}$ (such as the original table $G_{0}$ ) form the disclosure set $D S_{2}$, which clearly does not satisfy $(2,2)$-diversity, either.

The computation of disclosure sets has another complication as follows. Recall that to compute $D S_{i}$, we apply the algorithm $\mathbb{G}$ to each $X \in P E R\left(G_{i}\right)$. The algorithm $\mathbb{G}$ will then compute a disclosure set for each generalization $g_{j}(X)(1 \leq j \leq i-1)$. It may seem that we can then reuse previous results since the disclosure sets $g_{j}(X)(1 \leq$ $j \leq i-1$ ) should normally have been computed before we compute $D S_{i}$ (refer to the algorithm $\mathbb{G})$. However, this is not the case. The two sets $\operatorname{PER}\left(G_{i-1}\right)$ and $\operatorname{PER}\left(G_{i}\right)$ are generally not comparable. Some instance $X$ may appear in $\operatorname{PER}\left(G_{i}\right)$ (for example, $g_{2}(X)=g_{2}\left(G_{0}\right)$ ) but not in $\operatorname{PER}\left(G_{i-1}\right)$ (for example, $g_{1}(X) \neq g_{1}\left(G_{0}\right)$ ). For such an instance $X$, the disclosure sets for $g_{j}(X)(1 \leq j \leq i-1)$ must be computed from scratch. 


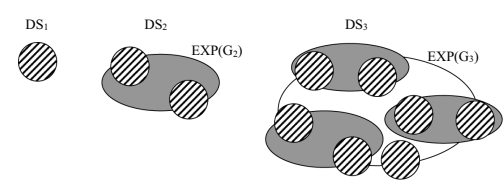

Fig. 1. Computing Disclosure Sets

Figure 1 illustrates this situation. The left-hand side denotes the disclosure set $D S_{1}$, which is equal to $\operatorname{EXP}\left(G_{1}\right)$. In the middle is $D S_{2}$ where the shaded oval represents $\operatorname{EXP}\left(G_{2}\right)$. Each of the two small circles denotes a set $P E R(X)$ that satisfies $C H K$ for some $X \in \operatorname{EXP}\left(G_{2}\right)$. All the instances in $\operatorname{EXP}\left(G_{2}\right) \backslash P E R(X)$ should thus be excluded from $D S_{2}$. Notice that while all instances in $\operatorname{PER}\left(G_{2}\right)$ yield the same generalization under $g_{2}$, they may yield different results under $g_{1}$, as indicated by the two disjoint circles (there may certainly be more than two different results under $g_{1}$ ). One subtlety here is that when we compute $D S_{2}$ we typically assume $D S_{1}$ does not satisfy $C H K$, so none of the small circles could be $D S_{1}$.

Example 3. In Table 2] any instance $X \in P E R\left(G_{2}\right)$ in which $E$ and $F$ are not both associated with $c_{4}$ will not appear in $\operatorname{PER}\left(G_{1}\right)$. The disclosure set $D S_{1}$ must thus be re-computed for each such $X$ while computing $D S_{2}$. On the other hand, for any such instance $X, P E R(X)$ will satisfy the $(2,2)$-diversity. If we represent those instances as small circles to be subtracted from $\operatorname{EXP}\left(G_{2}\right)$, as in Figure 1 there would be $3 \times 3-1=$ 8 such circles (in $\operatorname{PER}\left(G_{2}\right), E$ and $F$ can each be associated with three different values so totally nine different generalizations are possible under $g_{1}$ among which only $G_{1}$ does not satisfy the $(2,2)$-diversity).

The situation of computing $D S_{3}$ is similar but more complicated, as illustrated in the right-hand side of Figure 1 The ellipse depicts $P E R\left(G_{3}\right)$. We first consider how the algorithm $\mathbb{D S}$ will compute $D S_{3}$. For each $X \in P E R\left(G_{3}\right)$, the algorithm $\mathbb{G}$ may return $g_{1}$ if $C H K$ is satisfied on the disclosure set of $g_{1}(X)$ (that is, $P E R\left(g_{1}(X)\right)$ ), as represented by the small circle. If $C H K$ is not satisfied, the algorithm $\mathbb{G}$ will continue to compute the disclosure set for $g_{2}(X)$, which again involves computing a disclosure set for the generalization under $g_{1}$ on each instance in $P E R\left(g_{2}(X)\right)$. If $C H K$ is satisfied on the disclosure set of $g_{2}(X)$, then the algorithm $\mathbb{G}$ returns $g_{2}$. When $\mathbb{G}$ returns either $g_{1}$ or $g_{2}$, the algorithm $\mathbb{D S}$ will exclude the instance $X$ from $D S_{3}$. As illustrated in the right-hand side of Figure 1 an instance $X$ in $\operatorname{PER}\left(G_{3}\right)$ can satisfy one (and only one) of the following conditions.

1. $C H K$ holds on the disclosure set of $g_{1}(X)$ (illustrated as small circles in Figure 1)

2. $C H K$ holds on the disclosure set of $g_{2}(X)$ (illustrated as shaded areas)

3. $C H K$ does not hold on the disclosure set of $g_{1}(X)$ or $g_{2}(X)$ (illustrated as unfilled areas)

Example 4. In addition to the original table $G_{0}$ in Table 2, Table 4 shows two other possible instances in $\operatorname{PER}\left(G_{3}\right)$. The left-hand side table $G_{a}$ is an example of instances that 
satisfy the first condition since $\operatorname{PER}\left(g_{1}\left(G_{1}\right)\right)$ clearly satisfies $(2,2)$-diversity. Both the original table $G_{0}$ in Table 2 and the right-hand side table $G_{b}$ in Table 4 are examples of instances that satisfy the third condition.

In Example 4, although both $G_{0}$ in Table 2 and $G_{b}$ in Table 4 satisfy the third condition, they clearly do so in different ways. More specifically, $C H K$ does not hold on PER $\left(g_{1}\left(G_{b}\right)\right)$ or PER $\left(g_{2}\left(G_{b}\right)\right) ; C H K$ does not hold on PER $\left(G_{1}\right)$ but it does hold on $\operatorname{PER}\left(G_{2}\right)$. The reason that $G_{0}$ does not satisfy the second condition but the third is that $C H K$ does not hold on $D S_{2}$. Referring to Figure $1, P E R\left(g_{2}\left(G_{b}\right)\right)$ will be a shaded oval; $D S\left(G_{2}\right)$ will be a shaded oval (that is, $P E R\left(G_{2}\right)$ ) subtracted by some small circles (that is, $\operatorname{PER}\left(g_{1}(X)\right)\left(X \in P E R\left(G_{2}\right)\right)$ on which $C H K$ holds.

Table 4. Two Possible Instances in $\operatorname{PER}\left(G_{3}\right)$

\begin{tabular}{|c|c|c|c|c|}
\hline \multicolumn{2}{|c|}{ Table $G_{a}$} & \multirow{2}{*}{\multicolumn{3}{|c|}{ Table $G_{b}$}} \\
\hline$I D$ & \begin{tabular}{|l|l|}
$Q I$ & $S$ \\
\end{tabular} & & & \\
\hline$A$ & \begin{tabular}{|l|l|}
$g_{0}^{I}$ & $c_{1}$ \\
\end{tabular} & $A$ & & $c_{1}$ \\
\hline$B$ & \begin{tabular}{l|l}
$g_{0}^{2}$ & $c_{2}$
\end{tabular} & $B$ & $g_{0}^{2}$ & $c_{3}$ \\
\hline$C$ & \begin{tabular}{l|l}
$g_{0}^{3}$ & $c_{3}$
\end{tabular} & $C$ & $g_{0}^{3}$ & $c_{2}$ \\
\hline$D$ & \begin{tabular}{l|l}
$g_{0}^{4}$ & $c_{4}$ \\
\end{tabular} & $D$ & $g_{0}^{4}$ & $c_{2}$ \\
\hline$E$ & \begin{tabular}{l|l|l}
$g_{0}^{5}$ & $c_{2}$ \\
\end{tabular} & $E$ & $g_{0}^{5}$ & $c_{4}$ \\
\hline$F$ & \begin{tabular}{l|l}
$g_{0}^{6}$ & $c_{6}$
\end{tabular} & $F$ & $g_{0}^{6}$ & $c_{4}$ \\
\hline$G$ & \begin{tabular}{l|l}
$g_{0}^{7}$ & $c_{4}$ \\
\end{tabular} & $G$ & $g_{0}^{7}$ & $c_{6}$ \\
\hline$H$ & \begin{tabular}{|l|l|}
$g_{0}^{8}$ & $c_{5}$ \\
0
\end{tabular} & $H$ & $g_{0}^{8}$ & $c_{6}$ \\
\hline$I$ & \begin{tabular}{|l|l|}
$g_{0}^{9}$ & $c_{6}$
\end{tabular} & $I$ & $g_{0}^{9}$ & $c_{5}$ \\
\hline$J$ & $\begin{array}{ll}g_{0}^{10} & c_{7} \\
\end{array}$ & $J$ & & $c_{7}$ \\
\hline
\end{tabular}

We are now ready to consider which instances in $\operatorname{PER}\left(G_{3}\right)$ should be included in $D S_{3}$. Clearly, according to Definition 1 any instance that satisfies the first two conditions should be excluded, whereas instances satisfying the last condition should be included. Although the third condition can be satisfied in two different ways, we do not need to treat the two cases differently with the generalization algorithm $\mathbb{G}$ (however, we shall see the need for doing so in next section). In Figure $10 S_{3}$ corresponds to the unfilled area formed as the complement of all the small circles and shaded ovals.

Example 5. Both $G_{0}$ in Table 2 and $G_{b}$ in Table 4 will be included in $D S_{3}$, although they fail $(2,2)$-diversity in different ways (we shall see another case in next section).

In this special case, $D S_{3}$ can actually be computed more easily since there does not exist any $X \in \operatorname{PER}\left(G_{3}\right)$ that can satisfy the above second condition (that is, $(2,2)$ diversity is satisfied on the disclosure set of $g_{2}(X)$ ). Informally, any such $X$ must first allow the $(2,2)$-diversity to satisfy on $\operatorname{PER}\left(g_{2}(X)\right)$ but not on $\operatorname{PER}\left(g_{1}(X)\right.$ ) (for example, $G_{0}$ meets this requirement). However, we have that $g_{1}^{1}=g_{2}^{1}, g_{1}^{5}=g_{2}^{4}$, and 
$g_{2}^{2}$ and $g_{2}^{3}$ can satisfy $(2,2)$-diversity only if they each includes three different values. Therefore, the only possibility is that $g_{1}^{3}$ has two identical values, such as in the case with $G_{0}$. However, we already know that in this case the disclosure set of $g_{2}(X)$ will not satisfy $(2,2)$-diversity since both $E$ and $F$ must be associated with the same value. We conclude that the second condition cannot be satisfied by any instance in $\operatorname{PER}\left(G_{3}\right)$, and $D S_{3}$ can thus be computed by excluding from $\operatorname{PER}\left(G_{3}\right)$ any instance $X$ with $(2,2)$-diversity satisfied on $P E R\left(g_{1}(X)\right)$.

In Figure 1 a confusion may arise about the instances in $\operatorname{PER}\left(G_{i-1}\right) \backslash P E R\left(G_{i}\right)$, such as those inside the small circles but outside the shaded ovals. When we compute the disclosure set for $G_{2}$, for any instance $X \in P E R\left(G_{2}\right)$, we evaluate $C H K$ on the disclosure set of $g_{1}(X)$. It seems those instances in $\operatorname{PER}\left(g_{1}(X)\right) \backslash \operatorname{PER}\left(G_{2}\right)$ should be excluded during such an evaluation because we know those instances are not possible. However, this is not the case. The algorithm $\mathbb{D} \mathbb{S}$ simulates what an adversary will do to eliminate an instance $X$ from $\operatorname{PER}\left(G_{2}\right)$, he/she aims to prove that $X$ cannot be the original table. For this purpose, the adversary will first assume that $X$ is the original table and then attempt to show that $C H K$ is already satisfied on $\operatorname{PER}\left(g_{1}(X)\right)$. If this is indeed the case, then $g_{1}(X)$ would have been released, and thus the adversary would not have any knowledge about $g_{2}(X)$ at all.

\section{Exclusive Strategy}

The generalization algorithm $\mathbb{G}$ in Table 3 adopts a straightforward strategy in using the sequence of generalization functions $g_{1}, g_{2}, \ldots, g_{n}$. That is, each function is applied in the given order, and the first generalization whose disclosure set satisfies the privacy property will be returned. Although this strategy is a natural choice and has been adopted by most existing generalization algorithms, it is not necessarily the only choice, neither is it an optimal choice in terms of data utility or computational complexity. By adopting different strategies, we may develop different generalization algorithms from the same sequence of generalization functions. In this paper, we do not intend to give a comprehensive study of possible strategies. Instead, we only present one strategy that is more efficient and may lead to more data utility in some cases.

Recall that in Example 4, $G_{0}$ in Table 2 and $G_{b}$ in Table 4 are both included in $D S_{3}$. However, the difference lies in that $C H K$ does not hold on $\operatorname{PER}\left(g_{2}\left(G_{b}\right)\right)$ but it does on $\operatorname{PER}\left(G_{2}\right)$. An important observation is that we know $G_{b}$ should be included in $D S_{3}$ without computing any disclosure sets, whereas we do not know whether $G_{2}$ should be included in $D S_{3}$ until we compute $D S_{2}$ (and know it does not satisfy $C H K$ ). Such a recursive computation of $D S_{2}$ within that of $D S_{3}$ brings high complexity, and should be avoided if possible. We thus propose a different strategy in handling instances like $G_{0}$. That is, we simply do not include it in $D S_{3}$, regardless whether $D S_{2}$ satisfies $\mathrm{CHK}$ (notice that if $D S_{2}$ does satisfy $\mathrm{CHK}$ then $G_{2}$ will also be excluded from $D S_{3}$ ). If we were to represent this situation using Figure 1 then the shaded oval will correspond to any $\operatorname{PER}\left(g_{2}(X)\right)$ that satisfies $C H K$ (and the small circles remain to have the same meaning), regardless whether the corresponding disclosure set satisfies $C H K$. More generally, we exclude any instance $X \in \operatorname{EXP}\left(G_{i}\right)$ from $D S_{i}$, if only $\operatorname{EXP}\left(G_{j}\right)$ 
Table 5. Algorithms $\mathbb{G}_{e}$ and $\mathbb{D S}_{e}$
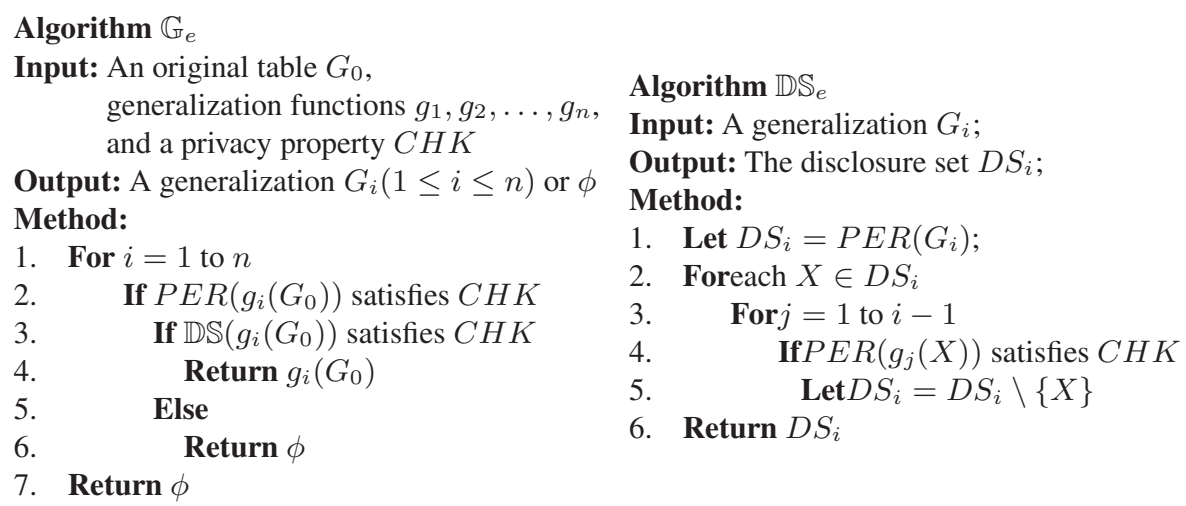

satisfies $C H K$. We present this exclusive strategy as Algorithm $\mathbb{G}_{e}$ in Table 5, On the other hand, we shall refer to Algorithm $\mathbb{G}$ in section 2 as the inclusive strategy from now on.

In Table 5, it can be noticed that both the algorithm for generalization and that for computing disclosure sets in the exclusive strategy are different from those in the inclusive strategy. This fact is a reflection of the inter-dependency between the two algorithms, or equivalently, the inter-dependency between the approach to generalization and adversary's knowledge. More specifically, the generalization algorithm $\mathbb{G}_{e}$ simply refuse to disclose anything, if the given original table yield a generalization $G_{i}$ for which $\operatorname{PER}\left(G_{i}\right)$ satisfies $C H K$ but $D S_{i}$ does not. An adversary also knows this fact since the algorithms are publicly known. In guessing the original table after seeing $G_{i}$ released, the adversary will test each instance $X \in P E R\left(G_{i}\right)$ to see whether $X$ can be the original table. However, different from the inclusive strategy, the exclusive strategy makes such a testing fairly simple. That is, any instance $X$ for which $\operatorname{PER}\left(g_{j}(X)\right)$ satisfies $C H K$ for some $j<i$ can be immediately eliminated from further consideration, because if $X$ were indeed the original table, then the algorithm $\mathbb{G}_{e}$ would have either returned $g_{j}(X)$ (if its disclosure set satisfies $C H K$ ) or nothing (if the disclosure set does not satisfy $C H K$ ) instead of releasing $G_{i}$.

Example 6. Consider applying the exclusive strategy to $G_{0}$ in Table 2 Clearly, the three generalizations $G_{1}, G_{2}$, and $G_{3}$ do not change, because we are still using the same generalization functions as before (but in a different way). The disclosure sets $D S_{1}$ and $D S_{2}$ also remain the same (note that $\operatorname{PER}\left(G_{1}\right)=D S_{1}$ ). When the algorithm $\mathbb{G}_{e}$ sees that $P E R\left(G_{1}\right)$ does not satisfy $C H K$, it continues to the next generalization function $g_{2}$ as with the inclusive strategy. However, when $\mathbb{G}_{e}$ sees $P E R\left(G_{2}\right)$ satisfies $C H K$ but $D S_{2}$ does not, it simply returns $\phi$ indicating that nothing can be disclosed (recall that with the inclusive strategy, $\mathbb{G}$ will continue to $\left.g_{3}\right)$. 
In contrast to the inclusive strategy, the exclusive strategy may seem to be a more drastic approach that may result in less data utility. Example 6 may seem to support this statement. However, this is in fact not the case. Due to space limitation, we cannot show $D S_{3}$ computed from Table 2 under the inclusive strategy, but we calculate the ratio of the association between $E$ and $c_{4}$ in Example 7

Example 7. As mentioned in Section 2.2 for this special case, $D S_{3}$ can be computed by excluding any instance $X$ for which $\operatorname{EXP}\left(g_{1}(X)\right)$ satisfies $C H K$. The instances in $D S_{3}$ must thus fall into following three sets. First, both $E$ and $F$ have $c_{4}$. Second, both $C$ and $D$ have $c_{2}$, and only one of $E$ and $F$ may have $c_{4}$ (the other will have $c_{6}$ ). Third, both $G$ and $H$ have $c_{6}$, and only one of $E$ and $F$ may have $c_{4}$ (the other will have $c_{2}$ ). These three sets are clearly disjoint. Moreover, by counting the number of permutations, we can see that the cardinality of the first set is $6 \times 2 \times 6=72(A, B$, and $C$ can have 6 different permutations; $D$ and $G$ can have 2, etc.) among which all have $E$ associated with $c_{4}$. Similarly, the second and third set each has $2 \times 6=12$ instances in which $E$ is associated with $c_{4}$, and the other 12 instances in which $E$ is associated with $c_{6}$ and $c_{2}$, respectively. We can thus conclude that the ratio of $E$ associated with $c_{4}$ is $(72+12+12) /(72+24+24)=0.8$.

By applying the inclusive strategy, the $(2,2)$-diversity is not satisfied on $D S_{3}$. Therefore, nothing can be disclosed under the inclusive strategy, either. That is, for the given original table $G_{0}$ (and also the substantialized table in Table 1), the two strategies yield the same data utility. Besides, there also exist other cases where the exclusive strategy will provide more data utility. Suppose now $G_{b}$ in Table 4 is given as the original table. Clearly, the inclusive strategy will disclose nothing because none of the generalizations through $G_{1}, G_{2}$ and $G_{3}$ can satisfy $(2,2)$-diversity. For exclusive strategy, neither $\operatorname{PER}\left(g_{1}\left(G_{b}\right)\right)$ nor $\operatorname{PER}\left(g_{2}\left(G_{b}\right)\right)$ can satisfy $(2,2)$-diversity. For $\operatorname{PER}\left(g_{3}\left(G_{b}\right)\right)$, again we calculate the ratio that $c_{4}$ is associated with $E$ among all conditions in Example 8.

Example 8. Following Example 7 $D S_{3}$ under the exclusive strategy can be obtained by eliminating any instance $X$ for which $P E R\left(g_{2}(X)\right)$ satisfy $(2,2)$-diversity from the previous result of $D S_{3}$ under the inclusive strategy. For the first set, $D$ and $G$ must now have $c_{2}$ and $c_{6}$, respectively, so we are left with 36 instances. Moreover, $C$ and $H$ must have 2 and 6 , respectively, leaving totally 20 instances all with $E$ associated with $c_{4}$. For the second and third set, nothing need to be eliminated. The ratio of $E$ associated with $c_{4}$ is thus now $(20+12+12) /(20+24+24)=0.647$. And this is also the maximal ratio of a single condition among all IDs.

Surprisingly, under the exclusive strategy, we can now disclose $G_{3}$ for the original table $G_{b}$ in Table 4 (a substantialized example is shown in Table 6. In another word, the exclusive strategy actually provides more data utility in this case. The reason lies in the fact that the privacy property (that is, $(2,2)$-diversity) is not set-monotonic [30], neither is the sequence of sets of possible instances $\operatorname{PER}\left(G_{i}\right)(i=1,2, \ldots, n)$. Generally, the data utility of the two strategies will be incomparable. Their performances depend on specific problem settings. 
Table 6. Another Example of Patient Information Table

\begin{tabular}{|c|c|c|}
\hline NAME & AGE & Condition \\
\hline Alice & 21 & flu \\
\hline Bob & 27 & pneumonia \\
\hline Clark & 31 & tracheitis \\
\hline Diana & 36 & tracheitis \\
\hline Ellen & 43 & gastritis \\
\hline Fen & 49 & gastritis \\
\hline George & 52 & cancer \\
\hline Henry & 58 & cancer \\
\hline Ian & 63 & enteritis \\
\hline Jason & 67 & heart disease \\
\hline
\end{tabular}

However, the exclusive strategy has an important advantage over the inclusive strategy, that is, a significantly lower complexity. In Table 5, unlike under the inclusive strategy, the algorithms under the exclusive strategy are not recursive because we do not call $\mathbb{G}_{e}$ within $\mathbb{D S}_{e}$. Denote $x_{i}$ the complexity for computing the disclosure set $D S_{i}$ under the inclusive strategy, and $y_{i}$ the cardinality of $\operatorname{PER}\left(G_{i}\right)$. We have that $x_{i}=\left(\sum_{j=1}^{i-1} x_{j}\right) \cdot y_{i}$ and $x_{1}=\left|G_{0}\right|$. By solving this recursive function, we can estimate the worst case complexity of the inclusive strategy to be $O\left(\left(\left|\operatorname{PER}\left(G_{\max }\right)\right|\right)^{n}\right)$ where $G_{\max }$ is a generalization with the maximum cardinality of possible instances. In contrast, the complexity of the exclusive strategy is $O\left(n^{2} \cdot\left|P E R\left(G_{\max }\right)\right|\right)$. By avoiding a recursive process, the exclusive strategy reduces the complexity from exponential to polynomial.

Other strategies are certainly possible, although their discussion is out of the scope of this paper. One complication is that the definition of disclosure sets given in Definition 1 should be generalized to accommodate the fact that the given sequence of generalization functions is not necessarily evaluated in the given order. The evaluation of those functions may actually happen in any order as defined in a strategy, and may vary depending on the given original table. For example, the exclusive strategy may directly jump to the last function (that returns $\phi$ ) from any step. One way to keep the Definition 1 valid in this particular case is to have multiple copies of the last function and place a copy in front of each generalization function in the given sequence. In each step, if the algorithm chooses to either return the current generalization or to use the copy of the last function to return $\phi$, then the current instance will be eliminated from the next disclosure set, which is in accordance with Definition 1

\section{Related Work}

Micro-data disclosure has been extensively studied [13 1016 17] where the security issue discussed in this paper is largely ignored. In particular, data swapping [9 23 28] and cell suppression [18] both aim to protect micro-data released in census tables. However, the amount of privacy is usually not measured in those earlier work. Miklau et. al presents an interesting measurement of information disclosed through tables based on 
the perfect secrecy notion by Shannon [8]. The important notion of $k$-anonymity is a model of privacy requirement [25] that received extensive studies in recent years. To achieve optimal $k$-anonymity (with the most utility) is shown to be computationally infeasible [21].

A model based on the intuition of blending individuals in a crowd is recently proposed in [27]. Personalized requirement for anonymity is studied in [29]. In [11], the authors approach the issue from a different perspective where the privacy property is based on generalization of the protected data and can be customized by users. Much efforts have been made around developing efficient $k$-anonymity algorithms

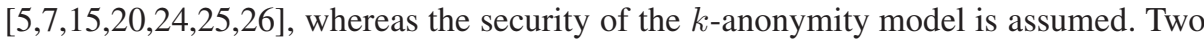
exceptions are the $l$-diversity notion proposed in [2] and the $t$-closeness notion proposed in [19], which address the deficiency of $k$-anonymity of allowing insecure groups with a small number of sensitive values. Algorithms developed for $k$-anonymity can be extended to $l$-diversity and $t$-closeness, but they still do not take into account an adversary's knowledge about generalization algorithms. In [30], the authors pointed out the above problem and proposed a model for the adversary's knowledge, but did not give any efficient solution for the general micro-data disclosure problem.

In contrast to micro-data disclosure, aggregation queries are the main concern in statistical databases [10:1322]. The main challenge is to answer aggregation queries without allowing an adversary to deduce secret individual values. The auditing methods in [6] address this problem by determining whether each new query can be safely answered based on previously answered queries. The authors of [6]12|14] consider the same problem in more specific settings of off-line auditing and online auditing, respectively. Closest to our work, the authors of [14] consider knowledge about the decision algorithm itself. However, it only applies to a limited case of aggregation queries and does not consider the current state of the database in determining the safety of a query.

\section{Conclusion}

Armed with knowledge about a generalization algorithm used for computing disclosed data, an adversary may deduce more information to violate a desired privacy property. We have studied this issue in the context of generalization-based micro-data disclosure algorithms. We showed that a naive solution to address this issue demands prohibitive computational cost. We then introduced an alternative exclusive strategy for generalization algorithms. Compare to the naive exponential algorithms based on the traditional inclusive strategy, algorithms based on exclusive strategy have much better efficiency (polynomial in the size of the table), and also, provide even better data utility in certain cases.

Acknowledgements. This material is partially supported by the National Science Foundation under grants CT-0716567, CT-0627493, IIS-0242237, and IIS-0430402; by the Army Research Office under the grant W911NF-07-1-0383; by the MITRE Technology Program; by the Natural Sciences and Engineering Research Council of Canada under Discovery Grant N01035; and by Fonds de recherche sur la nature et les technologies. The authors thank the anonymous reviewers for their valuable comments. 


\section{References}

1. Dobra, A., Feinberg, S.E.: Bounding entries in multi-way contingency tables given a set of marginal totals. In: Foundations of Statistical Inference: Proceedings of the Shoresh Conference 2000, Springer, Heidelberg (2003)

2. Machanavajjhala, A., Gehrke, J., Kifer, D., Venkitasubramaniam, M.: 1-diversity: Privacy beyond k-anonymity. In: Proceedings of the 22nd IEEE International Conference on Data Engineering (ICDE 2006) (2006)

3. Slavkovic, A., Feinberg, S.E.: Bounds for cell entries in two-way tables given conditional relative frequencies. Privacy in Statistical Databases (2004)

4. Dobkin, D.P., Jones, A.K., Lipton, R.J.: Secure databases: Protection against user influence. ACM TODS 4(1), 76-96 (1979)

5. Du, Y., Xia, T., Tao, Y., Zhang, D., Zhu, F.: On multidimensional k-anonymity with local recoding generalization

6. Chin, F.: Security problems on inference control for sum, max, and min queries. J.ACM 33(3), 451-464 (1986)

7. Aggarwal, G., Feder, T., Kenthapadi, K., Motwani, R., Panigrahy, R., Thomas, D., Zhu, A.: k-anonymity: Algorithms and hardness. Technical report, Stanford University (2004)

8. Miklau, G., Suciu, D.: A formal analysis of information disclosure in data exchange. In: SIGMOD (2004)

9. Duncan, G.T., Feinberg, S.E.: Obtaining information while preserving privacy: A markov perturbation method for tabular data. In: Joint Statistical Meetings, Anaheim, CA (1997)

10. Fellegi, I.P.: On the question of statistical confidentiality. Journal of the American Statistical Association 67(337), 7-18 (1993)

11. Byun, J., Bertino, E.: Micro-views, or on how to protect privacy while enhancing data usability: concepts and challenges. SIGMOD Record 35(1), 9-13 (2006)

12. Kleinberg, J., Papadimitriou, C., Raghavan, P.: Auditing boolean attributes. In: PODS (2000)

13. Schlorer, J.: Identification and retrieval of personal records from a statistical bank. Methods Info. Med. (1975)

14. Kenthapadi, K., Mishra, N., Nissim, K.: Simulatable auditing. In: PODS (2005)

15. LeFevre, K., DeWitt, D., Ramakrishnan, R.: Incognito: Efficient fulldomain k-anonymity. In: SIGMOD (2005)

16. Cox, L.H.: Solving confidentiality protection problems in tabulations using network optimization: A network model for cell suppression in the u.s. economic censuses. In: Proceedings of the Internatinal Seminar on Statistical Confidentiality (1982)

17. Cox, L.H.: New results in disclosure avoidance for tabulations. In: International Statistical Institute Proceedings (1987)

18. Cox, L.H.: Suppression, methodology and statistical disclosure control. J. of the American Statistical Association (1995)

19. Li, N., Li, T., Venkatasubramanian, S.: t-closeness: Privacy beyond k-anonymity and 1diversity. In: ICDE (2007)

20. Sweeney, L.: k-anonymity: a model for protecting privacy. International Journal on Uncertainty, Fuzziness and Knowledge-based Systems 10(5), 557-570 (2002)

21. Meyerson, A., Williams, R.: On the complexity of optimal k-anonymity. In: ACM PODS (2004)

22. Adam, N.R., Wortmann, J.C.: Security-control methods for statistical databases: A comparative study. ACM Comput. Surv. 21(4), 515-556 (1989)

23. Diaconis, P., Sturmfels, B.: Algebraic algorithms for sampling from conditional distributions. Annals of Statistics (1998) 
24. Samarati, P.: Protecting respondents identities in microdata release. In: IEEE TKDE, pp. 1010-1027 (2001)

25. Samarati, P., Sweeney, L.: Protecting privacy when disclosing information: k-anonymity and its enforcement through generalization and suppression. Technical report, CMU, SRI (1998)

26. Bayardo, R.J., Agrawal, R.: Data privacy through optimal k-anonymization. In: ICDE (2005)

27. Chawla, S., Dwork, C., McSherry, F., Smith, A., Wee, H.: Toward privacy in public databases. In: Theory of Cryptography Conference (2005)

28. Dalenius, T., Reiss, S.: Data swapping: A technique for disclosure control. Journal of Statistical Planning and Inference 6, 73-85 (1982)

29. Xiao, X., Tao, Y.: Personalized privacy preservation. In: SIGMOD (2006)

30. Zhang, L., Jajodia, S., Brodsky, A.: Information disclosure under realistic assumptions: Privacy versus optimality. In: ACM Conference on Computer and Communications Security (CCS) (2007) 